\title{
Dengue: Manifestações Cardíacas e Implicações na Terapêutica Antitrombótica
}

\author{
Dengue: Cardiac Manifestations and Implications in Antithrombotic Treatment \\ Antonio Eduardo Pesaro ${ }^{1,2}$, Élbio D'Amico 3 , Luis Fernando C. Aranha ${ }^{1,4}$ \\ Hospital Israelita Albert Einstein ${ }^{1}$ Instituto do Coração do Hospital das Clínicas ${ }^{2}$ e Hospital das Clínicas da FMUSP³ , Universidade Federal de São \\ Paulo - UNIFESP4 - São Paulo, SP - Brasil
}

\section{Introdução}

A dengue é causada por vírus do gênero Flavivirus, gênero esse composto por vírus RNA transmitidos, na maioria das vezes, por um artrópode, podendo causar doença em humanos. Outros vírus que causam doença de importância clínica são os da febre amarela e da encefalite japonesa ${ }^{1}$.

A dengue tem ampla distribuição em áreas tropicais, ocorrendo de forma endêmica e eventualmente epidêmica. É transmitida por mosquitos do gênero Aedes, principalmente Aedes aegypti, cuja distribuição e adaptação em ambientes urbanos e peridomiciliares explica a predominância da dengue em cidades. A doença ocorre com a introdução de paciente virêmico em ambiente onde há concentração suficiente de vetores, e torna-se epidêmica com grandes aumentos na concentração desses. O vírus subdivide-se em quatro sorotipos, que não conferem imunidade cruzada.

No Brasil, à semelhança de outros países da faixa tropical, a doença é endêmica com períodos epidêmicos. Neste ano, segundo dados do Ministério da Saúde, foram notificados, até 12 de março, 85.018 casos, representando um aumento de quase $30 \%$ em relação ao mesmo período do ano anterior. Os estados com maior número de casos são, pela ordem, Mato Grosso do Sul (50,4\%), Mato Grosso (7,2\%), Rio de Janeiro (5,2\%), Paraná (4,7\%), Minas Gerais $(4,6 \%)$ e São Paulo $(3,6 \%)$. Rio Grande do Sul e Santa Catarina são os únicos sem documentação de transmissão autóctone. Em razão da inespecificidade da apresentação clínica dos casos mais leves, esses números certamente são subestimados².

\section{Aspectos clínicos da dengue}

A maioria dos casos de dengue é autolimitada, cursando com quadro inespecífico de febre, mal-estar e fraqueza. Mais caracteristicamente, intensa dor muscular e cefaléia retro-ocular podem ocorrer, com ou sem rash cutâneo. Laboratorialmente, aumento de enzimas hepáticas, leucopenia e plaquetopenia são alterações compatíveis, mas não específicas da dengue ${ }^{1}$.

\section{Palavras-chave}

Dengue, febre hemorrágica da Dengue/complicações, arritmias, bloqueio atrioventricular, fibrinolíticos.

Correspondência: Antonio Eduardo Pesaro •

InCor - Av. Dr. Enéas de Carvalho Aguiar, 44 - 05403-000 - São Paulo, SP - Brasil E-mail: eduardopesaro@hotmail.com

Artigo recebido em 21/05/07, aceito em 21/05/07.
As formas mais graves incluem a síndrome do choque por dengue e a dengue hemorrágica. O choque é decorrente de importante alteração da permeabilidade capilar e grande extravasamento de plasma para sítios extravasculares, e está associado à ativação imune com elevados níveis séricos de receptor de fator de necrose tumoral- $\alpha$ (TNF), interleucina (IL)-8 e outros fatores.

A dengue hemorrágica desenvolve-se, em geral, em conjunto com choque e ocorre após período de dois a sete dias após defervescência. Clinicamente, não é possível distinguir aqueles pacientes que vão evoluir com a forma hemorrágica daqueles com manifestação autolimitada. No Brasil, este ano (de janeiro a maio), apenas 55 casos de dengue hemorrágica foram reportados, com seis óbitos².

\section{Fisiopatologia da dengue hemorrágica}

A dengue hemorrágica é manifestação rara e ocorre quase que exclusivamente em pacientes com experiência anterior, sugerindo fisiopatologia associada a hiper-resposta imune mediada por anticorpos heterólogos. Aumento de TNF, IL-2 e CD8 solúvel sugerem hiperativação de células CD4 e CD8 de memória. Há evidência de hiperexpressão de receptores Fc e antígenos MHC classes I e II e aumento sérico de vários mediadores inflamatórios como conseqüência de lise de células endoteliais e mononucleares. O resultante da hiperresposta imunológica é uma combinação de vasculopatia e coagulopatia de consumo. A diátese hemorrágica da dengue é causada por vasculopatia, trombocitopenia e coagulopatia leve, que são responsáveis pelos sangramentos cutâneos e mucosos $^{3}$. O aumento da fragilidade vascular é provavelmente decorrente da ação direta do vírus, que já ocorreria na fase de viremia ou estágio inicial febril.

\section{Plaquetopenia e plaquetopatia}

Quanto às plaquetas, pode haver plaquetopenia e plaquetopatia. A plaquetopenia pode ser secundária à menor produção medular de plaquetas e também da maior destruição periférica. Já se evidenciou que durante a fase aguda febril da dengue hemorrágica, a medula óssea apresenta-se acentuadamente hipocelular, com redução de todas as linhagens celulares. Esses achados foram, posteriormente, demonstrados como devidos à ação direta viral sobre as células do estroma medular e sobre as células progenitoras hematopoéticas. Dois dias antes do período de defervescência, observa-se hipercelularidade da medula óssea, com aumento das células precursoras das três linhagens 
celulares medulares. A presença de hemofagocitose pode ainda justificar a redução da contagem plaquetária, que também pode ocorrer por destruição imunológica (anticorpos antiplaquetários da classe IgM e anticorpos específicos contra a dengue). A contagem plaquetária retorna aos seus valores normais sete a dez dias após o período de defervescença ${ }^{4,5}$.

São ainda descritas alterações funcionais das plaquetas, evidenciadas através de hipoagregação induzida pelo ADP, com menor secreção de ADP intraplaquetário, e aumento das concentrações plasmáticas de betatromboglobulina e fator plaquetário 4. Esses achados são compatíveis com ativação plaquetária in vivo decorrente de ativação por imunocomplexos. A função plaquetária retorna às suas condições normais duas a três semanas após a fase inicial do período de convalescença ${ }^{5}$.

\section{Alterações da coagulação}

Durante o período febril, observam-se reduções variáveis da atividade de diferentes fatores da coagulação, como fibrinogênio, fator V, fator VII, fator VIII, fator IX e fator X, além da antitrombina e da $\alpha_{2}$-antiplasmina. Essas alterações justificam os prolongamentos discretos do tempo de protrombina e tempo de tromboplastina parcial ativada. São descritas elevações das concentrações dos produtos de degradação do fibrinogênio/fibrina (PDF) e dos dímeros-D ${ }^{5}$.

Em decorrência dessas alterações da hemostasia, o uso do ácido acetilsalicílico, de antiinflamatórios não-hormonais e administração de grandes quantidades de expansores de volume (Dextran 40 e Haemacel) são considerados fatores de risco para sangramentos ${ }^{6}$.

\section{Imunidade e inflamação}

Não são bem compreendidos os mecanismos responsáveis pelo desenvolvimento da doença hemorrágica grave, porém é aceito que uma infecção secundária por outro sorotipo seja o principal fator de risco para a ocorrência da dengue hemorrágica e da síndrome do choque da dengue. Anticorpos de uma infecção prévia com reatividade cruzada, mas não neutralizantes, ligam-se ao novo sorotipo, aumentando sua captação por monócitos e macrófagos, resultando em amplificação da cascata de citoquinas e ativação do complemento $^{7}$. Contudo, como somente de $2 \%$ a $4 \%$ dos indivíduos com uma segunda infecção desenvolvem doença grave, o incremento dependente de anticorpos não pode isoladamente explicar o processo total. Observam-se diferenças em anticorpos, citoquinas e respostas de células T entre pacientes com a forma não-complicada e aqueles com as formas complicadas.

Os monócitos, linfócitos B e mastócitos infectados pelo vírus da dengue produzem diferentes citoquinas, sendo demonstrado, por alguns autores, maiores aumentos das concentrações de TNF- $\alpha$, IL-2, IL- 6 e interferon (IFN)- $\gamma$ nos primeiros três dias de doença, com aparecimento posterior de IL-10, IL-5 e IL-4. Os pacientes com a forma hemorrágica apresentam maiores concentrações de TNF- $\alpha$, IL-6, IL-13, IL-18 e fator citotóxico, estando essas citoquinas implicadas no aumento da permeabilidade vascular e no choque que ocorrem durante a infecção. Ainda mais, o fator citotóxico, produzido células T CD4+, induz os macrófagos a produzirem IL-1 $\alpha$, TNF- $\alpha$ e IL-8. As concentrações de IL-6 (de origem endotelial e de mastócitos) são maiores nos pacientes com choque e dengue hemorrágica. Os níveis mais elevados de TNF- $\alpha$ e de IL-10 correlacionam-se com as manifestações hemorrágicas e plaquetopenia, respectivamente. A IL-10 também reduz a função plaquetária e contribui para os defeitos plaquetários da dengue ${ }^{4}$.

Existem muitas evidências interligando a inflamação com a coagulação, sendo as principais interfaces o fator tecidual (FT), o sistema da proteína C e o sistema fibrinolítico. Citocinas proinflamatórias podem afetar todos esses mecanismos da coagulação e proteases ativadas da coagulação, anticoagulantes fisiológicos ou componentes do sistema fibrinolítico podem modular a inflamação através de receptores celulares específicos.

Os principais mediadores inflamatórios envolvidos na ativação da coagulação são a IL-6, na ativação da coagulação, e o TNF- $\alpha$ e a IL-1 na regulação dos anticoagulantes fisiológicos. Vários estudos demonstram a importância da IL-6 na indução da expressão do FT por várias células, como as mononucleares, levando à ativação sistêmica da coagulação. Uma vez expresso o FT, é desencadeada a cascata da coagulação e a formação dos complexos enzimáticos que se formam sobre uma superfície fosfolipídica que, de maneira ideal, é apresentada pelas plaquetas. Durante o processo inflamatório as plaquetas podem ser diretamente ativadas por endotoxinas, pela trombina e por mediadores inflamatórios, como o fator ativador de plaquetas. As plaquetas ativadas ao expressar a P-seletina irão mediar a adesão plaquetária ao endotélio e leucócitos. Essa ligação das plaquetas ativadas aos neutrófilos e células mononucleares causa ativação do fator nuclear $\kappa \mathrm{B}$, aumentando a expressão de FT pelos monócitos. Durante o processo inflamatório grave, as concentrações de antitrombina estão marcantemente reduzidas por menor síntese, degradação por elastase dos neutrófilos ativados e consumo. O sistema da proteína C também é bloqueado, uma vez que a expressão endotelial da trombomodulina encontrase reduzida por ação do TNF- $\alpha$ e da IL-1 $\beta$. O TNF- $\alpha$ e a IL-1 $\beta$ também têm papel na regulação dos inibidores e ativadores do plasminogênio. As citoquinas promovem a secreção de tPA e uPA dos seus sítios de estoque nas células endotelais. Contudo, esse aumento da ativação fibrinolítica é contrabalançado por aumento retardado e mantido do PAI- ${ }^{7}$.

\section{Manifestações cardíacas da dengue}

O acometimento cardíaco pela dengue é considerado raro. Em 1996, durante uma epidemia na Índia, 206 pacientes foram avaliados e apenas um manifestou sintomas $\operatorname{cardía} \cos ^{8}$. Na literatura, há publicações de relatos de casos isolados de distúrbios de condução atrioventricular (ritmo juncional e bloqueio atrioventricular), arritmias supraventriculares e miocardites ${ }^{9}$.

Por sua vez, a disfunção ventricular associada à fase aguda da dengue hemorrágica foi descrita por diversos autores, e provavelmente é subdiagnosticada na prática clínica corriqueira. Durante a epidemia de 1996, 54 crianças com 
dengue e graus variados de severidade clínica foram submetidas à avaliação da função ventricular. Aproximadamente 16\% delas apresentaram fração de ejeção inferior a $50 \%{ }^{10}$. $\mathrm{Na}$ mesma ocasião, 17 indivíduos com dengue hemorrágica ou choque relacionado à dengue foram avaliados com cintilografia do miocárdio, e observou-se hipocinesia difusa em $70 \%$ dos pacientes, com fração de ejeção média ao redor de $40 \%{ }^{11}$. Após três semanas, todos os pacientes apresentaram normalização da função miocárdica. Mais recentemente, 24 pacientes com dengue hemorrágico foram avaliados e submetidos a ecocardiogramas seriados durante a infecção aguda e convalescença. No estudo, observou-se redução transitória da fração de ejeção ventricular e do índice cardíaco durante a infecção ${ }^{12}$.

Em conclusão, apesar de raramente haver manifestações cardíacas específicas na dengue, a depressão da função miocárdica é freqüente nos casos de doença hemorrágica ou choque associado. A "síndrome do choque relacionado à dengue" ocorre em razão do aumento da permeabilidade vascular e padrão hipovolêmico ${ }^{13}$. Entretanto, a adequada abordagem da instabilidade hemodinâmica relacionada à dengue requer, além de vigorosa expansão volêmica, avaliação e tratamento da disfunção ventricular associada, aos moldes do tratamento atual da sepse.

\section{Implicações da interrupção de antitrombóticos em cardiopatas com dengue}

A decisão de interromper antiagregantes plaquetários e anticoagulantes em pacientes com dengue depende de uma complexa avaliação de risco-benefício dessas terapêuticas. Devemos considerar paralelamente o risco da interrupção de antitrombóticos em diversos cenários clínicos, e o risco hemorrágico da infecção viral aguda.

Pacientes com angioplastias recentes, fibrilação atrial crônica (FAC) e portadores de próteses valvares metálicas são os que mais se beneficiam de antiagregantes e anticoagulantes, em longo prazo. A interrupção do tratamento promove incremento do risco trombótico de modo diferente em cada cenário clínico descrito a seguir.

Após a colocação de um stent, o tratamento com ácido acetilsalisílico (AAS) e tienopiridínicos (ticlopidina ou clopidogrel) é obrigatório e reduz drasticamente o risco de trombose aguda e subaguda intra-stent, além de eventos cardiovasculaes adversos ${ }^{14}$. Um estudo com 1.653 pacientes submetidos a angioplastia com stent evidenciou redução de 3,6\% para 0,5\% no risco de eventos adversos em 30 dias, quando tienopiridínicos foram associados ao $\mathrm{AAS}^{15}$. As recomendações atuais sugerem que, além do AAS, clopidogrel seja utilizado por no mínimo um mês após stents não-farmacológicos, três meses após stents revestidos com sirolimus, seis meses para plaxitel e, idealmente, 12 meses para todos, se não forem de alto risco hemorrágico ${ }^{16}$. Recentemente, a ocorrência de trombose tardia (após um ano) de stents farmacológicos levantou a discussão sobre a possibilidade de tratamento prolongado por tempo indeterminado com clopidogrel. Entretanto, a interrupção precoce de antiagregantes plaquetários, no primeiro mês após o tratamento com stents, pode ser devastadora, com incidência de até 30\% de trombose aguda ou subaguda intra-stent ${ }^{17}$.

Pacientes com FAC devem ser tratados com antiagregantes plaquetários ou anticoagulantes, para prevenção de trombose atrial e acidente vascular cerebral (AVC) cardioembólico. Os anticoagulantes devem ser preferidos naqueles pacientes com maior risco embólico (disfunção ventricular, idosos, hipertensos, diabéticos, valvopatas, ou com AVC prévio). Conforme a quantidade de fatores de risco, o risco anual de AVC em pacientes não anticoagulados pode variar entre $3 \% \mathrm{e}$ $20 \%{ }^{18}$. Uma metanálise demonstrou redução de $62 \%$ no risco de AVC com anticoagulantes em pacientes com FAC ${ }^{19}$.

Pacientes submetidos a implante de próteses valvares metálicas beneficiam-se de anticoagulação para prevenção de trombose valvar. Aqueles com próteses em posição mitral têm maior risco do que em posição aórtica. A presença de prótese de Starr-Edwards, antecedente de FAC, tromboembolismo prévio, mais de uma válvula mecânica e posição tricúspide também conferem maior risco trombótico. Entretanto, mesmo em pacientes com válvulas mecânicas, o risco de trombose anual sem a proteção da varfarina é de cerca de $20 \%{ }^{20}$. Portanto, a interrupção de varfarina por alguns dias não representa risco trombótico proibitivo.

\section{Conclusão e recomendações}

Em virtude da baixa incidência de dengue hemorrágica e da dificuldade de predizer, inicialmente, quais pacientes vão evoluir com quadro hemorrágico, podemos sugerir as seguintes medidas em relação à suspensão de antitrombóticos nas seguintes situações clínicas cardiológicas:

\section{1) Todos os pacientes com dengue}

O AAS deve ser evitado nos pacientes com Dengue por dois motivos:

O primeiro deles é a possibilidade da Síndrome de Reye. Apesar de rara, a síndrome de Reye, grave encefalopatia associada a hepatite, pode ser desencadeada por AAS em pacientes com diversas viroses, tais como varicela, influenza e dengue. Apesar de mais comum nas crianças, adultos também podem ser acometidos.

O segundo motivo é o risco de plaquetopenia pela dengue, que pode ser agravada pelo uso concomitante de antiagregantes plaquetários.

Recomendação: Todos os pacientes com dengue devem evitar AAS por uma semana, para redução do risco de síndrome de Reye e plaquetopenia grave. Em pacientes com alto risco de trombose, antiagregantes podem ser mantidos desde que as plaquetas sejam monitorizadas periodicamente (ver adiante).

2) Pacientes com dengue e alto risco de trombose em curto prazo

- Pacientes submetidos a angioplastia coronária com stents recentemente (um mês para não farmacológico, e três a seis meses para farmacológico).

- Portadores de próteses valvares mecânicas, 
particularmente em posição mitral, tricúspide, ou com FAC associada, tromboembolismo prévio ou mais de uma válvula mecânica.

- Portadores de FAC com múltiplos fatores de risco trombótico (disfunção ventricular, idosos, hipertensos, diabéticos, valvopatas, AVC prévio, trombo intracavitário).

Recomendação: Manter clopidogrel e AAS, naqueles que já recebiam. Suspender warfarina e substituir por heparina assim que INR estiver abaixo da faixa terapêutica. Reintroduzir varfarina após uma semana. Monitorizar seriadamente plaquetas e coagulograma até uma semana. Suspender as medicações, se contagem plaquetária igual ou inferior a $50.000 / \mathrm{mm}^{3}$, sangramento ou choque. Pode-se considerar a suspensão do clopidogrel e AAS de acordo com a intensidade da redução progressiva do número de plaquetas.

\section{Referências}

1. Tsai TF, Vaughn DW, Solomon T. Flaviviruses, In Mandell GL, Bennett JE, Dolin R (eds.). Principles and practice of infectious diseases. 6th ed. Philadelphia: Elsevier; 2005.

2. Brasil. Ministério da Saúde. Combate a dengue. [acesso em 2006 julho 10]. Disponível em URL: http://portal.saude.gov.br.

3. Isarangkura P, Mahasandana C, Chuansumrit A, Angchaisuksiri P. Acquired bleeding disorders: the impact of health problems in the developing world. Haemophilia. 2004; 10 (Suppl. 4): 188-95.

4. Malavige GN, Fernando S, Fernando DJ, Seneviratne SL. Dengue viral infections. Postgrad Med J. 2004; 80: 588-601.

5. Chuansumrit A, Tangnararatchakit K. Pathophysiology and management of dengue hemorrhagic fever. Transfusion Alternatives in Transfusion Medicine. 2006; 8 (Suppl 1): 3-11.

6. Wilder-Smith A, Schwartz E. Dengue in travelers. N Engl J Med. 2005; 353: 924-32.

7. Levi M, van der Poll T. Two-way interactions between inflammation and coagulation. Trends Cardiovasc Med. 2005; 15 (7): 254-9.

8. Agarwal R, Kapoor S, Nagar R, Misra A, Tandon R, Mathur A, et al. A clinical study of the patients with dengue hemorrhagic fever during the epidemic of 1996 at Lucknow, India. Southeast Asian J Trop Med Public Health. 1999; 30 (4):735-40

9. Khongphatthallayothin A, Chotivitayatarakorn P, Somchit S, Mitprasart A Sakolsattayadorn S, Thisyakorn C. Morbitz type I second degree AV block during recovery from dengue hemorrhagic fever. Southeast Asian J Trop Med Public Health. 2000; 31: 642-5.

10. Kabra SK, Juneja R, Madhulika JY, Singhal T, Dar L, Kothari SS, et al. Myocardial dysfunction in children with dengue haemorrhagic fever. Natl Med J India. 1998; 11: 59-61.

11. Wali JP, Biswas A, Chandra S, Malhotra A, Aggarwal P, Handa R, et al. Cardiac involvement in dengue haemorrhagic fever. Int J Cardiol. 1998; 64: 31-6.

12. Khongphatthanayothin $A$, SuesaowalakM, Muangmingsook S, Bhattarakosol P,Pancharoen C. Hemodynamic profiles of patients with dengue hemorrhagic fever during toxic stage: an echocardiographic study. Intensive Care Med. $2003 ;$ 29: 570-4
3) Pacientes com dengue e pequeno risco de trombose em curto prazo

- Portadores de doença arterial coronária estável.

- Pacientes submetidos a angioplastia coronária com stents há mais de seis meses.

- Portadores de FAC sem fatores de risco trombóticos (ou com um apenas).

- Portadores de prótese valvar biológica.

Recomendação: Suspender AAS. Considerar suspensão de clopidogrel e varfarina por uma semana.

\section{4) Pacientes com dengue hemorrágica}

Recomendação: Suspensão imediata de todos os antitrombóticos.

13. Kalayanarooj S, Vaughn DW, Nimmannitya S, Green S, Suntayakorn S Kunentrasai N. Early clinical and laboratory indicators of acute dengue illness. J Infect Dis. 1997; 176: 313-21.

14. Grines CL, Bonow RO, Casey DE Jr, Gardner TJ, Lockhart PB, Moliterno DJ, et al. Prevention of premature discontinuation of dual antiplatelet therapy in patients with coronary artery stents: a science advisory from the American Heart Association, American College of Cardiology, Society for Cardiovascular Angiography and Interventions, American College of Surgeons, and American Dental Association, with representation from the American College of Physicians. J Am Coll Cardiol 2007; 49:734-9.

15. Leon MB, Baim DS, Popma JJ, Gordon PC, Cutlip DE, Ho KK, et al. for the Stent Anticoagulation Restenosis Study Investigators. A clinical tria comparing three antithrombotic-drug regimens after coronary-artery stenting. N Engl J Med. 1998; 339: 1665-71.

16. Smith SC Jr, Feldman TE, Hirshfeld JW Jr, Jacobs AK, Kern MJ, King SB 3rd, et al. American College of Cardiology/American Heart Association Task Force of Practice Guidelines, ACC/AHA/SCAI Writing Committee to Update the 2001 Guidelines for Percutaneous Coronary Intervention. Circulation. 2006; 113 (7): e166-286.

17. lakovou I, Schmidt T, Bonizzoni E, Sangiorgi GM, Stankovic G, Airoldi F, et al. Incidence, predictors, and outcome of thrombosis after successful implantation of drug-eluting stents. JAMA. 2005; 293 (17): 2126-30.

18. van Walraven WC, Hart RG, Wells GA, Wells GA, Petersen P, Koudstaal PJ, et al. A clinical prediction rule to identify patients with atrial fibrillation and a low risk for stroke while taking aspirin. Arch Intern Med. 2003;163 (8): 936-43.

19. Hart RG, Benavente O, McBride R, Pearce LA. Antithrombotic therapy to prevent stroke in patients with atrial fibrillation: a meta-analysis. Ann Intern Med. 1999; 131 (7): 492-501.

20. Bonow RO, Carabello BA, Kanu C, de Leon AC Jr, Faxon DP, Freed MD, et al. ACC/AHA 2006 guidelines for the management of patients with valvular heart disease: a report of the American College of Cardiology/American Heart Association Task Force on Practice Guidelines Circulation. 2006; 114: e84-231. 